\title{
UNIVERSITY OF BONN NATURAL RADIOCARBON MEASUREMENTS III
}

H. W. SCHARPENSEEL and F. PIETIG

Institut für Bodenkunde, Universität Bonn

Bonn, Bundesrepublik Deutschland

The laboratory has continued to concentrate on soil and water dating, using the benzene method as outlined in Scharpenseel and Pietig (1969a).

\section{ACKNOWLEDGMENTS}

Much of the technical work for sample preparation was carried out by $\mathrm{E}$. Kruse and $\mathrm{H}$. Topüth. We are indepted to $\mathrm{G}$. Strunk-Lichtenberg for help in the preparation of a computer program to facilitate calculation of results. This work was supported by grants from the German Federal Department of Scientific Research. Preparation of carbonate samples from aquifers in Tunisia was financed by the German Federal Department of Economic Cooperation.

\section{SAMPLE DESCRIPTIONS \\ I. GROUND WATER SAMPLES \\ A. Cologne 07 sand aquifer}

After 2 yr, a 3 rd repetition of radiocarbon measurements was made on same wells of the Cologne 07 sand aquifer, reported previously (Radiocarbon, 1968 , v. 10, p. 8-28 and Radiocarbon, 1969, v. 11, p. 3-14). This is a continuing study of subterranean water movement (Tamers, Balke, and Scharpenseel, 1969) based on nuclear-weapon-produced excesses of $\mathrm{C}^{14}$, whose variation over the past decade is measured, Radiocarbon, 1969, v. 11, p. 10-13. Carbonates were extracted by the method of Tamers (1967). Tritium concentrations are also measured. Samples coll. 1969 and subm. by members of Radiocarbon Dating Lab.

\begin{tabular}{ccc}
\hline & & $\begin{array}{c}\mathrm{C}^{14} \text { age, } \\
\text { corrected } \\
\text { according } \\
\text { to Tamers } \\
(1967)\end{array}$ \\
\hline $\begin{array}{c}\text { BONN-572. } \\
\left(51^{\circ} 1^{\prime} \text { N Lat, } 6^{\circ} 44^{\prime} \text { E Long) }\right.\end{array}$ & $\begin{array}{c}\mathrm{C}^{14} \text { age } \\
\text { uncorrected }\end{array}$ & $\begin{array}{c}\text { Modern } \\
\text { BONN-573. Widdersdorf } \\
\quad\left(50^{\circ} 58^{\prime} \text { N Lat, } 6^{\circ} 50^{\prime} \text { E Long) }\right.\end{array}$ \\
$\begin{array}{c}\text { BONN-574. Dansweiler } \\
\left(50^{\circ} 57^{\prime} \text { N Lat, } 6^{\circ} 46^{\prime} \text { E Long) }\right.\end{array}$ & $6540 \pm 45$ & $5290 \pm 415$ \\
$\begin{array}{c}\text { BONN-575. Königsdorf } \\
\left(50^{\circ} 56^{\prime} \text { N Lat, } 6^{\circ} 46^{\prime} \text { E Long) }\right.\end{array}$ & $6825 \pm 90$ & $5435 \pm 465$
\end{tabular}




\begin{tabular}{|c|c|c|}
\hline Sample & $\begin{array}{c}\mathrm{C}^{14} \text { age } \\
\text { uncorrected }\end{array}$ & $\begin{array}{l}\mathrm{C}^{14} \text { age, } \\
\text { corrected } \\
\text { according } \\
\text { to Tamers } \\
\quad(1967)\end{array}$ \\
\hline $\begin{array}{l}\text { BONN-576. Glessen } \\
\left(50^{\circ} 58^{\prime} \text { N Lat, } 6^{\circ} 45^{\prime} \text { E Long }\right)\end{array}$ & $1510 \pm 70$ & $\begin{array}{r}260 \pm 415 \\
\text { (Modern) }\end{array}$ \\
\hline $\begin{array}{l}\text { BONN-577. Synthern } \\
\left(50^{\circ} 58^{\prime} \text { N Lat, } 6^{\circ} 47^{\prime} \text { E Long }\right)\end{array}$ & $1735 \pm 50$ & $\begin{array}{r}390 \pm 445 \\
\text { (Modern) }\end{array}$ \\
\hline $\begin{array}{l}\text { BONN-578. Buschbell } \\
\left(50^{\circ} 56^{\prime} \text { N Lat, } 6^{\circ} 48^{\prime} \text { E Long }\right)\end{array}$ & $6440 \pm 65$ & $5640 \pm 330$ \\
\hline \multicolumn{3}{|c|}{$\begin{array}{l}\text { Comment: movement of water fairly slow, compared to distance of } \\
\text { piezometric tubes from which samples are taken. For more reliable flow } \\
\text { speed measurement another series of radiocarbon and tritium results must } \\
\text { be produced } 2 \text { or } 3 \text { yr later. Preliminary estimate of flow velocity is } \\
\text { aggravated by apparent artificial disturbances of flow direction. }\end{array}$} \\
\hline
\end{tabular}

\section{B. Netherrhine series}

Samples are dated in support of thesis work (Balke, 1969) on distribution, flow velocity, and recharge of ground water aquifers in the Netherrhine. Samples coll. 1969 and subm. by K. D. Balke, Geol. Landesamt Northrhine Westfalia, Krefeld, and members of Radiocarbon Dating Lab.

BONN-225. Etgendorf $2307 / 4$ $\left(50^{\circ} 59^{\prime} \mathrm{N}\right.$ Lat, $6^{\circ} 33^{\prime} \mathrm{E}$ Long)

BONN-226. Etgendorf $2307 / 3$ $\left(50^{\circ} 59^{\prime} \mathrm{N}\right.$ Lat, $6^{\circ} 33^{\prime} \mathrm{E}$ Long)

BONN-227. Etgendorf $2307 / 2$ $\left(50^{\circ} 59^{\prime} \mathrm{N}\right.$ Lat, $6^{\circ} 33^{\prime} \mathrm{E}$ Long)

BONN-228. Etgendorf $2307 / 1$ $\left(50^{\circ} 59^{\prime} \mathrm{N}\right.$ Lat, $6^{\circ} 33^{\prime} \mathrm{E}$ Long)

BONN-516. Margaretenhof $\left(50^{\circ} 57^{\prime} \mathrm{N}\right.$ Lat, $6^{\circ} 25^{\prime} \mathrm{E}$ Long)

BONN-517. B 32 Rheinbraun $\left(50^{\circ} 58^{\prime} \mathrm{N}\right.$ Lat, $6^{\circ} 37^{\prime}$ E Long)

BONN-518. MT 36 $\left(50^{\circ} 52^{\prime} \mathrm{N}\right.$ Lat, $6^{\circ} 44^{\prime} \mathrm{E}$ Long)

BONN-519. Oberembt $\left(50^{\circ} 57^{\prime} \mathrm{N}\right.$ Lat, $6^{\circ} 30^{\prime} \mathrm{E}$ Long)

BONN-520. Pegel 4.141, 4 Blatzheim $\left(50^{\circ} 51^{\prime} \mathrm{N}\right.$ Lat, $6^{\circ} 36^{\prime} \mathrm{E}$ Long)

$$
\begin{gathered}
10,100 \pm 95 \\
8150 \text { в.C. } \\
14,200 \pm 100 \\
12,250 \text { в.C. } \\
4200 \pm 60 \\
2250 \text { в.C. } \\
4720 \pm 80 \\
2770 \text { в.C. } \\
1530 \pm 70 \\
\text { A.D. } 420 \\
4020 \pm 50 \\
2070 \text { в.C. } \\
970 \pm 60 \\
\text { A.D. } 980 \\
2800 \pm 80 \\
850 \text { в.C. } \\
2640 \pm 80 \\
690 \text { в.C. }
\end{gathered}
$$


Comment: dates help to confirm identity of aquifers feeding different wells. BONN-227, -228 , and -517 as well as BONN-516, -518 and BONN$519,-520$ belong together. BONN-226 overlies -225 and was expected to be younger. Dates BONN-225, -226 indicate, that aquifer from BONN. 225 must have lateral connection with younger water resources, or BONN. 226 is alimented by older fossil water reserves.

\section{Landesbad Aachen wells}

Wells belong to area of medical bathing resort. Identity of new wells with those already exploited is confirmed by chemical analysis and $\mathrm{C}^{14}$ dating. Samples coll. 1968 and subm. by Prof. Schuler, Landesbad Aachen.

BONN-509. Landesbad Aachen-Burscheidt, Quelle A $14,200 \pm 205$ $\left(50^{\circ} 47^{\prime} \mathrm{N}\right.$ Lat, $6^{\circ} 4^{\prime} \mathrm{E}$ Long) 12,250 B.c.

BONN-510. Landesbad Aachen-Burscheidt, Quelle D $\quad 11,570 \pm 90$ $\left(50^{\circ} 47^{\prime} \mathrm{N}\right.$ Lat, $6^{\circ} 4^{\prime} \mathrm{E}$ Long) 9620 B.c.

BONN-511. Landesbad Aachen-Burscheidt, Tiefenquelle $17,140 \pm 225$ $\left(50^{\circ} 47^{\prime} \mathrm{N}\right.$ Lat, $6^{\circ} 4^{\prime} \mathrm{E}$ Long) 15,190 B.c.

\section{HOAG/Ruhrchemie $/ R W W$ series}

Samples stem from aquifer of limited extent. Industries with increasing water consumption need information on extent of recharge. Also tritium concentrations are measured. Samples coll. 1968 and subm. by members of Radiocarbon Dating Lab. B.P. dates are after bicarbonate correction according to Tamers (1967).

BONN-513. Brunnen IV, Franz Haniel (HOAG) (51 $1^{\circ} 33^{\prime} \mathrm{N}$ Lat, $6^{\circ} 53^{\prime}$ E Long)

$$
\begin{gathered}
11,290 \pm 155 \\
9340 \text { в.c. } \\
9650 \pm 570 \text { в.P. } \\
4490 \pm 80 \\
2540 \text { в.C. } \\
2875 \pm 540 \text { в.P. } \\
9370 \pm 100 \\
7420 \text { в.c. } \\
7640 \pm 590 \text { в.P. }
\end{gathered}
$$

BONN-514. RWW, Rhein, Westf. Wasserwerke (51 ${ }^{\circ} 32^{\prime} \mathrm{N}$ Lat, $6^{\circ} 49^{\prime}$ E Long)

BONN-515. Ruhrchemie

$\left(51^{\circ} 31^{\prime} \mathrm{N}\right.$ Lat, $6^{\circ} 48^{\prime} \mathrm{E}$ Long)

Comment: increase in age from $\mathrm{E}$ to $\mathrm{W}$. As presumed, some recharge from E fringes. Abrupt drop in age of BONN-514 due to past break-in of younger water through demolished pit mouth.

\section{E. Venezuela water sample, Meachiche}

A portion of the sample was previously dated at $10,730 \pm 120$ B.P. (Tamers, 1966; IVIC-218). Coll. 1966 and subm. by M. A. Tamers, IVIC, Caracas, as check sample. 
BONN-512. Meachiche, Venezuela 8530 в.C.

(11 $1^{\circ} 20^{\prime} \mathrm{N}$ Lat, $69^{\circ} 34^{\prime} \mathrm{W}$ Long)

Comment: agrees with Venezuela IVIC measurement within $1 \sigma$ error range.

\section{F. Tunisia series}

As a lst sample series within 3-yr project of dating some of Tunisia's subterranean water reserves, carbonates of 76 wells were collected. $\mathrm{C}^{\mathbf{1 4}}$ ages are indicated, uncorrected and corrected for dead carbonate-C contibution (Tamers, 1967). In all samples also tritium concentrations are measured. Samples coll. 1968 and subm. by H. W. Scharpenseel and H. Gewehr, Inst. für Bodenkunde, Bonn Univ., J. Ohling, HER-Economic Cooperation Project, Tunis.

BONN-229. Kairouan II (35 $40^{\prime} \mathrm{N}$ Lat, $10^{\circ} 05^{\prime} \mathrm{E}$ Long)

BONN-230. Kairouan III ( $35^{\circ} 40^{\prime} \mathrm{N}$ Lat, $10^{\circ} 5^{\prime} \mathrm{E}$ Long)

BONN-231. El Grine V (35 $36^{\prime} \mathrm{N}$ Lat, $9^{\circ} 52^{\prime} \mathrm{E}$ Long)

BONN-232. El Grine II (35 $36^{\prime} \mathrm{N}$ Lat, 9 $9^{\circ} 52^{\prime} \mathrm{E}$ Long)

BONN-233. El Haouareb ( $35^{\circ} 34^{\prime} \mathrm{N}$ Lat, $9^{\circ} 45^{\prime}$ E Long)

BONN-234. Bled Sbitha (353 $31^{\prime} \mathrm{N}$ Lat, $9^{\circ} 49^{\prime}$ E Long)

BONN-235. Sidi Ali Ben Salem (35 $33^{\prime} \mathrm{N}$ Lat, $9^{\circ} 54^{\prime} \mathrm{E}$ Long)

BONN-236. Zafrana IV (35 $32^{\prime} \mathrm{N}$ Lat, $10^{\circ} 4^{\prime} \mathrm{E}$ Long)

BONN-237. Puit Zafrana IV (35 $31^{\prime} \mathrm{N}$ Lat, $10^{\circ} 4^{\prime} \mathrm{E}$ Long)

BONN-238. Sidi Amor Ben Hadjla ( $35^{\circ} 23^{\prime} \mathrm{N}$ Lat, $10^{\circ} 2^{\prime} \mathrm{E}$ Long)

BONN-239. Bir Boussari (35 $23^{\prime} \mathrm{N}$ Lat, $9^{\circ} 55^{\prime} \mathrm{E}$ Long)

BONN-240. Bir Djeclid (35 $24^{\prime} \mathrm{N}$ Lat, $9^{\circ} 56^{\prime} \mathrm{E}$ Long)

\begin{tabular}{c} 
Measured age \\
\hline $14,090 \pm 150$ \\
12,140 в.C. \\
$24,300 \pm 500$ \\
22,350 в.C. \\
$5570 \pm 50$ \\
3620 в.C. \\
$3070 \pm 50$ \\
1120 в.C. \\
$5030 \pm 40$ \\
3080 в.C. \\
$5590 \pm 60$ \\
3640 в.C. \\
$8460 \pm 50$ \\
6510 в.C. \\
$22,490 \pm 370$ \\
20,540 в.C. \\
$3160 \pm 75$ \\
1210 в.C. \\
$21,390 \pm 150$ \\
19,440 в.C. \\
$9790 \pm 140$ \\
7840 в.C. \\
$7805 \pm 105$ \\
5855 в.C. \\
190
\end{tabular}

Corrected age

$12,470 \pm 540$ 10,520 B.C.

$$
21,820 \pm 830
$$$$
19,870 \text { в.C. }
$$

$3380 \pm 720$

1430 B.C.

$1310 \pm 560$ A.D. 640

$2930 \pm 700$ 980 B.C.

$3630 \pm 650$ 1680 B.C.

$6140 \pm 770$ 4190 B.C.

$21,150 \pm 450$ 19,200 B.C.

Modern

$19,860 \pm 510$ 17,910 B.C.

$8450 \pm 440$ 6500 B.C.

$6400 \pm 480$ 4450 в.C. 
BONN-241. Puit Boussari

(352 23' N Lat, 9० $56^{\prime}$ E Long)

BONN-242. Zafrana 4 $\left(35^{\circ} 30^{\prime} \mathrm{N}\right.$ Lat, $10^{\circ} 8^{\prime} \mathrm{E}$ Long)

BONN-243. Ain El Bell

$\left(35^{\circ} 31^{\prime} \mathrm{N}\right.$ Lat, $10^{\circ} 12^{\prime} \mathrm{E}$ Long)

BONN-244. Draa el Oust $\left(35^{\circ} 40^{\prime} \mathrm{N}\right.$ Lat, $10^{\circ} 10^{\prime} \mathrm{E}$ Long)

BONN-245. Bir Naceur Chaffra $\left(35^{\circ} 41^{\prime} \mathrm{N}\right.$ Lat, $10^{\circ} 10^{\prime} \mathrm{E}$ Long)

BONN-246. Puit Service Foret (35 $26^{\prime} \mathrm{N}$ Lat, $9^{\circ} 50^{\prime} \mathrm{E}$ Long)

BONN-247. Draa Chouk (35 $45^{\prime} \mathrm{N}$ Lat, $10^{\circ} 08^{\prime} \mathrm{E}$ Long)

BONN-248. El Goutass I $\left(35^{\circ} 37^{\prime} \mathrm{N}\right.$ Lat, $9^{\circ} 56^{\prime} \mathrm{E}$ Long)

BONN-249. Kairouan IIb (3539' $\mathrm{N}$ Lat, $10^{\circ} 6^{\prime} \mathrm{E}$ Long)

BONN-250. Puits Membetch III (35 $37^{\prime} \mathrm{N}$ Lat, $9^{\circ} 55^{\prime} \mathrm{E}$ Long)

BONN-251. Bir Romani I (35 $38^{\prime} \mathrm{N}$ Lat, $10^{\circ} 6^{\prime} \mathrm{E}$ Long)

BONN-252. Bir Hadj Sadok (35 $24^{\prime} \mathrm{N}$ Lat, $9^{\circ} 53^{\circ} \mathrm{E}$ Long)

BONN-253. El Khadra (35 $29^{\prime} \mathrm{N}$ Lat, $10^{\circ} 1^{\prime} \mathrm{E}$ Long)

BONN-254. Zafrana III (35 $27^{\circ} \mathrm{N}$ Lat, $10^{\circ} 4^{\prime} \mathrm{E}$ Long)

BONN-255. Sidi Ahmed (35 $25^{\prime} \mathrm{N}$ Lat, $10^{\circ} 5^{\prime} \mathrm{E}$ Long)

BONN-256. Pavillier (35 25' N Lat, 9० $51^{\prime}$ E Long)

BONN-257. Draa Tammar I (35 $45^{\prime} \mathrm{N}$ Lat, $10^{\circ} 5^{\prime} \mathrm{E}$ Long)

BONN-258. Draa Tammar II (35 $43^{\prime} \mathrm{N}$ Lat, $10^{\circ} 5^{\prime} \mathrm{E}$ Long)
Measured age

$1100 \pm 30$
A.D. 850
$13,830 \pm 80$
11,880 B.C.

$$
\begin{aligned}
& 2370 \pm 50 \\
& 420 \text { B.C. }
\end{aligned}
$$

$21,240 \pm 310$

19,290 B.C.

$14,320 \pm 135$

12,370 B.C.

$$
3940 \pm 30
$$

1990 B.C.

$29,260 \pm 370$

27,310 B.C.

$9245 \pm 40$

7295 B.C.

$13,550 \pm 150$

11,600 B.C.

$4000 \pm 40$

2050 B.C.

$4790 \pm 90$

2840 B.C.

$6200 \pm 60$

4250 B.C.

$6530 \pm 80$

4580 B.C.

$15,620 \pm 80$

13,670 в.с.

$$
11,470 \pm 90
$$

9520 B.C.

$4200 \pm 80$

2250 B.C.

$16,230 \pm 430$

14,280 B.C.

$19,850 \pm 110$

17,900 B.C.
Corrected age

Modern

$12,530 \pm 430$

10,580 B.C.

A.D. 970

$980 \pm 480$

$20,190 \pm 350$

18,240 B.C.

$12,930 \pm 480$

10,980 B.C.

$2170 \pm 580$

220 B.C.

$27,960 \pm 430$

26,010 B.c.

$7905 \pm 450$

5955 B.c.

$12,250 \pm 430$

10,300 B.C.

$$
\begin{aligned}
& 2230 \pm 590 \\
& 280 \text { в.C. }
\end{aligned}
$$

$$
3400 \pm 460
$$

1450 B.C.

$4570 \pm 540$

2620 B.C.

$5090 \pm 480$

3140 B.C.

$14,380 \pm 415$

12,430 B.C.

$10,030 \pm 480$

8080 B.C.

$2130 \pm 690$

180 B.C.

$14,980 \pm 420$

13,030 B.C.

$18,550 \pm 430$

16,600 B.C. 


\begin{tabular}{|c|c|c|}
\hline 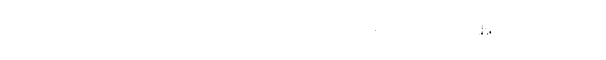 & Measured age & Corrected age \\
\hline $\begin{array}{l}\text { BONN-259. El Goutass III } \\
\left(35^{\circ} 37^{\prime} \mathrm{N} \text { Lat, } 10^{\circ} 1^{\prime} \mathrm{E} \text { Long }\right)\end{array}$ & $\begin{array}{l}9660 \pm 70 \\
7710 \text { в.с. }\end{array}$ & $\begin{array}{l}7860 \pm 600 \\
5910 \text { в.C. }\end{array}$ \\
\hline $\begin{array}{l}\text { BONN-260. Sidi Amor Ben Hadjla I } \\
\left(35^{\circ} 23^{\prime} \text { N Lat, } 10^{\circ} 3^{\prime} \text { E Long }\right)\end{array}$ & $\begin{array}{l}11,260 \pm 100 \\
9310 \text { в.c. }\end{array}$ & $\begin{array}{l}10,520 \pm 245 \\
8570 \text { в.с. }\end{array}$ \\
\hline $\begin{array}{l}\text { BONN-261. Sbiba } 11 \\
\quad\left(35^{\circ} 31^{\prime} \text { N Lat, } 9^{\circ} 4^{\prime} \text { E Long }\right)\end{array}$ & $\begin{array}{l}2600 \pm 70 \\
650 \text { в.с. }\end{array}$ & Modern \\
\hline $\begin{array}{l}\text { BONN-262. Sbiba } 12 \\
\left(35^{\circ} 31^{\prime} \text { N Lat, } 9^{\circ} 3^{\prime} \text { E Long }\right)\end{array}$ & $\begin{array}{l}7170 \pm 80 \\
5220 \text { в.с. }\end{array}$ & $\begin{array}{l}4410 \pm 920 \\
2460 \text { B.c. }\end{array}$ \\
\hline $\begin{array}{l}\text { BONN-263. Sbiba } 5 \\
\left(35^{\circ} 31^{\prime} \text { N Lat, } 9^{\circ} 2^{\prime} \text { E Long }\right)\end{array}$ & $\begin{array}{l}3110 \pm 80 \\
2160 \text { в.с. }\end{array}$ & $\begin{array}{l}1340 \pm 580 \\
\text { A.D. } 610\end{array}$ \\
\hline $\begin{array}{l}\text { BONN-264. Kasserine } 14 \\
\left(35^{\circ} 8^{\prime} \mathrm{N} \text { Lat, } 8^{\circ} 50^{\prime} \text { E Long }\right)\end{array}$ & $\begin{array}{l}3960 \pm 40 \\
2010 \text { в.C. }\end{array}$ & $\begin{array}{l}2570 \pm 460 \\
620 \text { в.c. }\end{array}$ \\
\hline $\begin{array}{l}\text { BONN-265. Ain Alouche } \\
\left(35^{\circ} 10^{\prime} \text { N Lat, } 8^{\circ} 48^{\prime} \text { E Long }\right)\end{array}$ & $\begin{array}{l}9250 \pm 90 \\
7300 \text { в.C. }\end{array}$ & $\begin{array}{l}5650 \pm 1200 \\
3700 \text { в.c. }\end{array}$ \\
\hline $\begin{array}{l}\text { BONN-266. } \\
\left(35^{\circ} 9^{\prime} \text { N Lasserine } 11\right. \\
\left.8^{\circ} 48^{\prime} \text { E Long }\right)\end{array}$ & $\begin{array}{l}10,470 \pm 150 \\
8520 \text { в.с. }\end{array}$ & $\begin{array}{l}8750 \pm 570 \\
6800 \text { в.с. }\end{array}$ \\
\hline $\begin{array}{ll}\text { BONN-267. } & \text { Kasserine } 12 \\
\left(35^{\circ} 9^{\prime} \mathrm{N}\right. & \left.\text { Lat, } 8^{\circ} 48^{\prime} \text { E Long }\right)\end{array}$ & $\begin{array}{l}13,820 \pm 70 \\
11,870 \text { в.с. }\end{array}$ & $\begin{array}{l}12,090 \pm 575 \\
10,140 \text { в.C. }\end{array}$ \\
\hline $\begin{array}{l}\text { BONN-268. Tozeur Gare } \\
\left(33^{\circ} 55^{\prime} \text { N Lat, } 8^{\circ} 8^{\prime} \text { E Long }\right)\end{array}$ & $\begin{array}{l}8280 \pm 180 \\
6330 \text { в.C. }\end{array}$ & $\begin{array}{l}6380 \pm 630 \\
4430 \text { в.c. }\end{array}$ \\
\hline $\begin{array}{l}\text { BONN-269. Sebaa Biar } \\
\left(34^{\circ} 0^{\prime} \text { N Lat, } 8^{\circ} 14^{\prime} \text { E Long }\right)\end{array}$ & $\begin{array}{l}16,450 \pm 240 \\
14,500 \text { в.с. }\end{array}$ & $\begin{array}{l}14,250 \pm 730 \\
12,300 \text { в.C. }\end{array}$ \\
\hline $\begin{array}{l}\text { BONN-270. Seddada } \\
\left(34^{\circ} 1^{\prime} \text { N Lat, } 8^{\circ} 17^{\prime} \text { E Long }\right)\end{array}$ & $\begin{array}{l}18,490 \pm 430 \\
16,540 \text { в.с. }\end{array}$ & $\begin{array}{l}16,730 \pm 590 \\
14,780 \text { в.с. }\end{array}$ \\
\hline $\begin{array}{l}\text { BONN-271. Puits Haffa } \\
\left(33^{\circ} 55^{\prime} \text { N Lat, } 8^{\circ} 8^{\prime} \text { E Long }\right.\end{array}$ & $\begin{array}{l}133.5 \pm 0.4 \% \\
\text { Modern }\end{array}$ & Modern \\
\hline $\begin{array}{l}\text { BONN-272. } \quad \text { Nefta } 3 \\
\left(33^{\circ} 52^{\prime} \text { N Lat, } 7^{\circ} 52^{\prime} \text { E Long }\right)\end{array}$ & $\begin{array}{l}11,050 \pm 175 \\
9100 \text { в.с. }\end{array}$ & $\begin{array}{l}9470 \pm 530 \\
7520 \text { B.C. }\end{array}$ \\
\hline $\begin{array}{l}\text { BONN-273. El Hamma } 8 \\
\left(34^{\circ} 0^{\prime} \mathrm{N} \text { Lat, } 8^{\circ} 10^{\prime} \text { E Long }\right)\end{array}$ & $\begin{array}{l}13,880 \pm 100 \\
11,930 \text { в.С. }\end{array}$ & $\begin{array}{l}12,030 \pm 610 \\
10,080 \text { в.C. }\end{array}$ \\
\hline $\begin{array}{l}\text { BONN-274. Gouifla } \\
\left(34^{\circ} 13^{\prime} \text { N Lat, } 8^{\circ} 12^{\prime} \text { E Long }\right)\end{array}$ & $\begin{array}{l}14,970 \pm 560 \\
13,020 \text { в.с. }\end{array}$ & $\begin{array}{l}13,290 \pm 560 \\
11,340 \text { в.C. }\end{array}$ \\
\hline $\begin{array}{l}\text { BONN-275. Mnagaa (Gafsa) } \\
\left(34^{\circ} 24^{\prime} \text { N Lat, 8 } 8^{\circ} 48^{\prime} \text { E Long) }\right.\end{array}$ & $\begin{array}{l}10,370 \pm 60 \\
8420 \text { в.c. }\end{array}$ & $\begin{array}{l}9690 \pm 230 \\
7740 \text { в.с. }\end{array}$ \\
\hline $\begin{array}{l}\text { BONN-276. Sidi Mansour } \\
\left(34^{\circ} 25^{\prime} \text { N Lat, } 8^{\circ} 48^{\prime} \text { E Long }\right)\end{array}$ & $\begin{array}{l}12,680 \pm 100 \\
10,730 \text { в.C. }\end{array}$ & $\begin{array}{l}11,190 \pm 490 \\
9240 \text { в.с. }\end{array}$ \\
\hline
\end{tabular}




\section{BONN-277. El Guettar (34 $20^{\prime} \mathrm{N}$ Lat, $8^{\circ} 54^{\prime} \mathrm{E}$ Long) \\ BONN-278. Seftimi 1

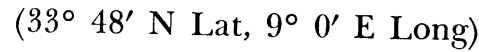 \\ BONN-279. Tombar 3 \\ (33 $44^{\prime} \mathrm{N}$ Lat, $8^{\circ} 53^{\prime} \mathrm{E}$ Long) \\ BONN-280. Douz}

(33 $26^{\prime} \mathrm{N}$ Lat, $9^{\circ} 1^{\prime}$ E Long)

BONN-281. Chenchou

(335 $54^{\prime}$ N Lat, $9^{\circ} 52^{\prime}$ E Long)

BONN-282. Gabés ICN 3

(33' $59^{\prime} \mathrm{N}$ Lat, $10^{\circ} 2^{\prime} \mathrm{E}$ Long)

BONN-283. Mareth I b (33 $37^{\prime} \mathrm{N}$ Lat, $9^{\circ} 50^{\prime} \mathrm{E}$ Long)

BONN-284. Dakhlet et Bibane (30 $26^{\prime} \mathrm{N}$ Lat, $9^{\circ} 53^{\prime} \mathrm{E}$ Long)

BONN-285. Tiaret SP 3 $\left(30^{\circ} 58^{\prime} \mathrm{N}\right.$ Lat, $10^{\circ} 8^{\prime} \mathrm{E}$ Long)

BONN-286. Bir Oulet Lorzet ( $31^{\circ} 46^{\prime} \mathrm{N}$ Lat, $10^{\circ} 20^{\prime} \mathrm{E}$ Long)

BONN-287. Fort Saint (30 ${ }^{\circ} 45^{\prime} \mathrm{N}$ Lat, $9^{\circ} 32^{\prime} \mathrm{E}$ Long)

BONN-288. Zarzis $\left(33^{\circ} 29^{\prime} \mathrm{N} \mathrm{Lat}, 11^{\circ} 4^{\prime} \mathrm{E}\right.$ Long)

BONN-289. Qualegh (Djerba) $\left(33^{\circ} 53^{\prime} \mathrm{N}\right.$ Lat, $10^{\circ} 59^{\prime} \mathrm{E}$ Long)

BONN-290. El Djazira (Djerba) (335 $51^{\prime} \mathrm{N}$ Lat, $10^{\circ} 58^{\prime} \mathrm{E}$ Long)

BONN-291. Sfax Siap (34 $43^{\prime} \mathrm{N}$ Lat, $10^{\circ} 46^{\prime} \mathrm{E}$ Long)

BONN-292. Oued Sohil (36 $31^{\prime} \mathrm{N}$ Lat, $10^{\circ} 42^{\prime} \mathrm{E}$ Long)

BONN-293. Oued Sidi Youssef ( $36^{\circ} 46^{\prime} \mathrm{N}$ Lat, $10^{\circ} 6^{\prime} \mathrm{E}$ Long)

BONN-294. Taffeloun

$\left(36^{\circ} 41^{\prime} \mathrm{N}\right.$ Lat, $10^{\circ} 53^{\prime}$ E Long)
Measured age

$14,900 \pm 150$

12,950 B.C.

$17,210 \pm 460$

15,260 в.C.

$12,550 \pm 90$

10,600 в.C.

$9960 \pm 60$

8010 B.C.

$12,110 \pm 100$

10,160 в.C.

$13,170 \pm 350$

11,220 B.C.

$11,620 \pm 80$

9670 B.C.

$14,520 \pm 80$

12,570 в.C.

$16,140 \pm 200$

14,190 в.c.

$10,950 \pm 60$

9000 B.c.

$13,060 \pm 150$

11,110 B.C.

$10,830 \pm 100$

8880 B.C.

$2870 \pm 90$

920 B.C.

$21,330 \pm 160$

19,380 B.c.

$23,900 \pm 250$

21,950 B.C.

$10,920 \pm 130$

8970 B.C.

$103.5 \pm 0.7 \%$

Modern

$4540 \pm 60$ 2590 B.C.
Corrected age

$13,320 \pm 530$

11,370 B.C.

$16,260 \pm 320$

14,310 B.C.

$9670 \pm 820$

7720 в.C.

$9010 \pm 315$

7060 B.c.

$10,860 \pm 420$

8910 B.C.

$12,020 \pm 380$

10,070 B.c.

$10,620 \pm 330$

8670 B.C.

$13,080 \pm 480$

11,130 B.C.

$14,840 \pm 43 \mathrm{C}$

12,890 B.C.

$9950 \pm 330$

8000 B.c.

$$
11,620 \pm 480
$$

9670 B.C.

$8230 \pm 860$

6280 B.C.

A.D. 1210

$740 \pm 710$

$19,430 \pm 630$ 17,480 в.C.

$20,880 \pm 1005$ 18,930 в.c.

$9670 \pm 420$

7720 в.C.

Modern

$$
3240 \pm 450
$$

1290 B.C. 


\begin{tabular}{|c|c|c|}
\hline & $\mathrm{Me}$ & Corrected age \\
\hline $\begin{array}{l}\text { ONN-295. Dar Chichou } 9447 \\
\left(36^{\circ} 58^{\prime} \text { N Lat, } 10^{\circ} 57^{\prime} \text { E Long }\right)\end{array}$ & & $\begin{array}{l}220 \\
\text { c. }\end{array}$ \\
\hline $\begin{array}{l}\text { BONN-296. Dar Chichou } 8303 \\
\left(37^{\circ} 0^{\prime} \mathrm{N} \text { Lat, } 10^{\circ} 27^{\prime} \text { E Long }\right)\end{array}$ & & 445 \\
\hline $\begin{array}{l}\text { BONN-297. Ain Taho } \\
\left(36^{\circ} 15^{\prime} \text { N Lat, } 9^{\circ}\right.\end{array}$ & $\begin{array}{l}7230 \pm \\
5280 \mathrm{~B}\end{array}$ & 490 \\
\hline $\begin{array}{l}\text { BONN-298. } \quad \text { Tabarka } 1 \\
\left(36^{\circ} 55^{\prime} \mathrm{N} \text { Lat, } 8^{\circ} 39^{\prime} \text { E Long }\right)\end{array}$ & $\begin{array}{l}1240 \pm 70 \\
\text { A.D. } 710\end{array}$ & $\begin{array}{l}380 \pm 350 \\
\text { A.D. } 1570\end{array}$ \\
\hline $\begin{array}{l}\text { BONN-299. Bulla Regia } \\
\left(36^{\circ} 33^{\prime} \text { N Lat, } 8^{\circ} 45^{\prime} \text { E Long }\right)\end{array}$ & $\begin{array}{r}950 \pm 60 \\
\text { A.D. } 1000\end{array}$ & Modern \\
\hline $\begin{array}{l}\text { BONN-300. Ain Beida } \\
\left(36^{\circ} 13^{\prime} \text { N Lat, } 8^{\circ} 56^{\prime} \text { E Long }\right)\end{array}$ & $\begin{array}{l}3990= \\
2040 \text { в }\end{array}$ & $\begin{array}{l}2050 \pm 640 \\
100 \text { B.C. }\end{array}$ \\
\hline $\begin{array}{l}\text { BONN-501. Le Kef } 4 \\
\left(36^{\circ} 9^{\prime} \text { N Lat, } 8^{\circ} 42^{\prime} \text { E Long }\right)\end{array}$ & $\begin{array}{l}2100 \pm 30 \\
150 \text { в.C. }\end{array}$ & $\begin{aligned} & 770 \pm 440 \\
& \text { A.D. } 1180\end{aligned}$ \\
\hline $\begin{array}{l}\text { BONN-502. Bled Abida } \\
\left(36^{\circ} 0^{\prime} \mathrm{N} \text { Lat, } 8^{\circ} 46^{\prime} \text { E Long }\right)\end{array}$ & 7950 & $\begin{array}{l}=650 \\
\text { c. }\end{array}$ \\
\hline $\begin{array}{l}\text { BONN-503. Ebba Ksour } \\
\left(35^{\circ} 59^{\prime} \quad \text { N Lat, } 8^{\circ} 49^{\prime} \text { E Long }\right)\end{array}$ & $\begin{array}{l}15,520= \\
13,570 \mathrm{r}\end{array}$ & $\begin{array}{l}13,920 \pm 530 \\
11,970 \text { в.С. }\end{array}$ \\
\hline $\begin{array}{c}\text { ONN-521. S. Amor Sidi } \\
\text { Bou Hadjla } \\
\left(35^{\circ} 22^{\prime} \text { N Lat, } 10^{\circ} 4^{\prime} \text { E Long }\right)\end{array}$ & $\begin{array}{l}16,700 \pm 120 \\
14,750 \text { в.с. }\end{array}$ & $\begin{array}{l}15,500 \pm 380 \\
13,550 \text { в.с. }\end{array}$ \\
\hline
\end{tabular}

Comment: BONN-229-260 and BONN-521 are from Kairouan area, captured in the Quaternary and Pliocene, BONN-261-267 from the SbibaKasserine zone in Miocene sandstone and limestone, BONN-268-277, N of Schott el Djerid in Mio-Pliocene, BONN-278-287, E of Djerid and extreme $\mathrm{S}$ in Cretaceous (esp. Cenomanian and Turonian), BONN 288290 at Djerba I. and neighboring Zarzis in Oligo-Miocene and PlioQuaternary, BONN-292-296 on Cap Bon Peninsula in Pliocene (esp. Astian and Plaisancian), and BONN-297-300 as well as BONN-501-503 from Medjerdah valley in Campanian, Quaternary, Eocene and Jurassic. Samples came from water holes and from wells, both artesian and ordinary. After 2nd and 3rd yr results of sample series are available, aquifers with modern recharge and those with "fossil" water only (without alimentation) will be listed, to assist systematic water management. Further isochrones will be drawn to connect wells of equal age and chemical composition.

\section{SOIL SAMPLES}

Soil samples were freed from roots and organic debris as already described in Radiocarbon, 1968, v. 10, p. 8-28; 1969, v. 11, p. 3-14. Carbon 
analysis was carried out by method of Rauterberg and Kremkus (1951). Fractionation of soil organic matter followed the basic procedure by Flaig, Scheffer, and Klamroth (1955) in slight modification (Scharpenseel, Ronzani, and Pietig, 1968).

\section{A. Chernozem and Steppe soils}

Organic material of fossil A horizon (fA) in B horizon of Parabraunerde (hapludalf) in wall of clay pit.

BONN-403. Parabraunerde with fossil chernozem Lantershofen, $0.8 \% \mathrm{C}, \mathrm{A}_{\mathrm{p}} 10$ to $20 \mathrm{~cm}$

$$
\begin{array}{r}
60 \pm 30 \\
\text { A.D. } 1890
\end{array}
$$

$$
\begin{array}{r}
980 \pm 60 \\
\text { A.D. } 970
\end{array}
$$
Lantershofen, $0.8 \% \mathrm{C}, \mathrm{A}_{1} 25$ to $30 \mathrm{~cm}$

$$
3550 \pm 50
$$$$
1600 \text { B.c. }
$$

Lantershofen, $1.8 \% \mathrm{C}, \mathrm{fAB}_{\mathrm{t} 1} 45$ to $55 \mathrm{~cm}$

BONN-406. Parabraunerde with fossil chernozem Lantershofen, $0.8 \% \mathrm{C}, \mathrm{fAB}_{\mathrm{t} 2} 55$ to $75 \mathrm{~cm}$

BONN-407. Parabraunerde with fossil chernozem Lantershofen, $0.7 \% \mathrm{C}, \mathrm{fAB}_{\mathrm{t} 3} 75$ to $95 \mathrm{~cm}$

BONN-408. Charcoal under disturbed chernozem humus (fossil) in Parabraunerde Lantershofen, 60 to $65 \mathrm{~cm}$

BONN-409. Humic horizon at 150 to $170 \mathrm{~cm}$, containing $1500 \pm 60$ charcoal, bones, and pieces of brick, $\quad$ A.D. 450 Lantershofen

Samples belong to different genetic horizons of Parabraunerde (hapludalf) profile on Würm loess with $\mathrm{fA}$ material of chernozem in present day $\mathbf{B}_{t}$ $\left(\mathrm{B}_{2}\right)$ horizon., clay pit Lantershofen (50 $33.5^{\prime} \mathrm{N}$ Lat, $7^{\circ} 7^{\prime} \mathrm{E}$ Long). Coll. 1968 and subm. by E. Kopp and H. W. Scharpenseel. Comment: BONN-403 -407 indicate presence of fossil $A$ horizon within $B_{t}\left(B_{2}\right)$ horizon of profile, maximum age $5530 \mathrm{yr}$, such as observed in modern chernozems (BONN-105, BONN-112, BONN-113). Charcoal of BONN408 and BONN-409 are too young to be in undisturbed position.

Fossil chernozem (paleudoll) buried under trachyt pumice.

BONN-411. Ochtendung, direct. Plaidt, Sample Michelsberg I, under disturbed trachyt pumice (fine roots), $0.5 \% \mathrm{C}, 120$ to $140 \mathrm{~cm}$

BONN-412. Same location, $0.3 \% \mathrm{C}, 140$ to $160 \mathrm{~cm}$ $5850 \pm 70$ 3900 B.c. $6990 \pm 80$ 5040 в.с.

BONN-413. Between Ochtendung and Plaidt, Sample Michelsberg II. Under slope cover of halfweathered pumice, few fine roots penetrate, $10,580 \pm 100$ upper $15 \mathrm{~cm}$ in 3 to $4 \mathrm{~m}$ depth, $0.6 \% \mathrm{C}$ 
BONN-414. Same location, lower $15 \mathrm{~cm}, 0.4 \% \mathrm{C}$

$$
\begin{gathered}
10,060 \pm 100 \\
8110 \text { в.C. } \\
10,020 \pm 90 \\
8070 \text { в.C. }
\end{gathered}
$$

BONN-415. $3 \mathrm{Km} \mathrm{S}$ Ochtendung, in direction of

Koblenz, digging deeper at fresh cut in

trachyt pumice cover, $0.3 \% \mathrm{C}, 260$ to $275 \mathrm{~cm}$

BONN-416. Same location, $0.3 \% \mathrm{C}, 275$ to $290 \mathrm{~cm}$

$$
\begin{gathered}
10,230 \pm 120 \\
8280 \text { в.C. }
\end{gathered}
$$

Samples are from slightly darker fossil chernozem A horizon, forming upper layer of Würm loess. On top cover of trachyt pumice. Samples BONN-411-414 Michelsberg, between Ochtendung and Plaidt (Rhineland Pfalz) $\left(50^{\circ} 21^{\prime} \mathrm{N}\right.$ Lat, $7^{\circ} 19^{\prime} \mathrm{E}$ Long), Samples $415-416$ in fresh pumice pit $3 \mathrm{~km} \mathrm{~S}$ Ochtendung, next to road in direction of Koblenz $\left(50^{\circ} 20^{\prime} \mathrm{N}\right.$ Lat, $7^{\circ} 18^{\prime} \mathrm{E}$ Long). Coll. 1968 and subm. by E. Kopp and H. W. Scharpenseel. Comment: samples underlie layer of trachyt pumice, spread in its present position by Aller $\phi \mathrm{d}$ volcanism. Estimated minimum age: 10 to $11,000 \mathrm{yr}$. Few visible deep-reaching roots cause slight rejuvenation. Ages are about twice as high as BONN-407, whose origin is in same humic horizon in unburied site. With necessary caution correction factor for rejuvenation in recent German chernozem profiles could be estimated to be ca. 2 .

Fossil chernozem $\mathrm{fA}$ in Parabraunerde (hapludalf) $\mathrm{B}_{\mathrm{t}}\left(\mathrm{B}_{2}\right)$ horizon.

BONN-417. Degraded fossil chernozem in Parabraunerde, $540 \pm 60$ E rim, clay pit Muddersheim, Rhineland, A.D. 1410 $1.34 \% \mathrm{C}, \mathrm{A}_{\mathrm{h}}, 0$ to $30 \mathrm{~cm}$

$750 \pm 50$

BONN-418. Same location, $0.58 \% \mathrm{C}, \mathrm{A}_{1}, 30$ to $70 \mathrm{~cm} \quad$ A.D. 1200

BONN-419. Same location, $0.54 \% \mathrm{C}, \mathrm{fAB}_{\mathrm{t} 1}, 70$ to $90 \mathrm{~cm}$ A.D. 350

$1600 \pm 50$

$2660 \pm 50$

BONN-420. Same location, $0.43 \% \mathrm{C}, \mathrm{fAB}_{\mathrm{t} 2}, 90$ to $120 \mathrm{~cm} \quad 710$ B.C.

BONN-421. Same location, $0.48 \% \mathrm{C}, \mathrm{fAB}_{\mathrm{v}}, 120$ to $165 \mathrm{~cm} \quad 1750$ B.C.

Samples of Parabraunerde profile (hapludalf) in Würm loess with darker B horizons, Muddersheim/Rhineland, "Muddersheimer Kumm", E fringe of clay pit $\left(50^{\circ} 45^{\prime} \mathrm{N}\right.$ Lat, $6^{\circ} 39^{\prime} \mathrm{E}$ Long). Coll. 1968 and subm. by G. Strunk-Lichtenberg of the Institute (Strunk-Lichtenberg, 1968). Comment: in $\mathrm{B}_{\mathrm{t} 1}$ horizon fragment of string ceramics found and archeologically dated to 3000 to 3500 B.c. This again would indicate a rejuvenation factor for $\mathbf{B}_{\mathrm{t} 1}$ horizon of ca. 2 (see preceding series). Dark color and abrupt step up of age in $B_{t}(B$,$) horizon (Bonn-419-421) suggests$ humic material of fossil chernozem (fA) in argillic and $B_{v}$ horizon. 

soils.

Humic matter containing Würm loess with buried fossil steppe

BONN-422. Fossil steppe soil, Quarry Schäferkalkwerke $\left(50^{\circ} 19^{\prime} \mathrm{N}\right.$ Lat, $8^{\circ} 4^{\prime} \mathrm{E}$ Long)

$21,430 \pm 220$ $0.3 \% \mathrm{C}, 560$ to $585 \mathrm{~cm}$

BONN-423. Fossil steppe soil, Gravel Pit E Weilbach (50॰ $3^{\prime} \mathrm{N}$ Lat, $8^{\circ} 6^{\prime} \mathrm{E}$ Long) $0.2 \% \mathrm{C}, 640$ to $680 \mathrm{~cm}$ 19,480 B.C.

$23,100 \pm 300$ 21,150 B.C.

BONN-424. Fossil steppe soil, Gravel Pit E Weilbach (50 $3^{\prime} \mathrm{N}$ Lat, $8^{\circ} 6^{\prime} \mathrm{E}$ Long) $0.2 \% \mathrm{C}, 580$ to $620 \mathrm{~cm}$

$17,950 \pm 375$ 16,000 B.c.

BONN-425. Fossil steppe soil, Gravel Pit SO Weilbach $\left(50^{\circ} 3^{\prime} \mathrm{N}\right.$ Lat, $8^{\circ} 6^{\prime} \mathrm{E}$ Long) $0.4 \% \mathrm{C}, 340$ to $360 \mathrm{~cm}$

$19,680 \pm 180$

17,730 в.с.

BONN-426. Fossil steppe soil, Tilery OB 45 HanauRossdorf, (50 $11^{\prime} \mathrm{N}$ Lat, $8^{\circ} 15^{\prime} \mathrm{E}$ Long) $0.4 \% \mathrm{C}, 350$ to $370 \mathrm{~cm}$

$17,000 \pm 570$ 15,050 B.C.

BONN-427. Fossil steppe soil, Dyckerhoff-Quarry, Wiesbaden, $\left(50^{\circ} 3^{\prime} \mathrm{N}\right.$ Lat, $8^{\circ} 17^{\prime} \mathrm{E}$ Long) $0.4 \% \mathrm{C}, 600$ to $690 \mathrm{~cm}$

$25,000 \pm 700$ 2350 B.C.

BONN-428. Fossil steppe soil, Dyckerhoff-Quarry $20,720 \pm 520$ Wiesbaden, $\left(50^{\circ} 3^{\prime} \mathrm{N}\right.$ Lat, $8^{\circ} 17^{\prime} \mathrm{E}$ Long) $0.2 \% \mathrm{C}, 470$ to $530 \mathrm{~cm}$

18,770 в.с.

BONN-429. Fossil steppe soil, Dyckerhoff-Quarry, $23,770 \pm 470$ Wiesbaden, (50 $3^{\prime} \mathrm{N}$ Lat, $8^{\circ} 17^{\prime} \mathrm{E}$ Long) $0.4 \% \mathrm{C}, 400$ to $440 \mathrm{~cm}$

21,820 B.C.

BONN-430. Fossil steppe soil, Dyckerhoff-Quarry, $20,550 \pm 180$ Wiesbaden, $\left(50^{\circ} 3^{\prime} \mathrm{N}\right.$ Lat, $8^{\circ} 17^{\prime} \mathrm{E}$ Long) 18,600 B.c. $0.2 \% \mathrm{C}, 230$ to $270 \mathrm{~cm}$

BONN-431. Fossil steppe soil, Tilery Wallertheim $21,380 \pm 490$ (49 $2^{\prime} \mathrm{N}$ Lat, $8^{\circ} 3^{\prime} \mathrm{E}$ Long) $0.5 \%$ N, $300 \mathrm{~cm} \quad 19,430$ в.C.

Samples of layered fossil soils, taken in various spots of dark steppe soil area Rhine-Pfalz and Rhine-Hessen. Samples serve to elucidate questions of Quarternary stratigraphy and chronology of fossil steppe soil formations. Coll. 1968 and subm. by A. Semmel, Hessisches Landesamt für Bodenforschung, Wiesbaden. Comment: samples BONN-422-431 were expected to stem from Old Würm with ages beyond 35,000 yr. Further samples will be measured.

Various horizons of soil profiles from Boehmen and Maehren (Czechoslovakia). Scrutiny of different dark soils, such as chernozems (hapludoll) on loess and marl, pseudogley chernozem (haplaquoll), smonitza (vertisol)like chernozems. 
BONN-437. (Boehmen, chernozem lessivé, Griserde from loess, Kozojedy, Jicin Dist., $1210 \pm 50$ $\left(50^{\circ} 19^{\prime} \mathrm{N}\right.$ Lat, $15^{\circ} 21^{\prime}$ E Long) $1.5 \% \mathrm{C}, \mathrm{A}_{\mathrm{p}}, 0$ to $20 \mathrm{~cm}$

BONN-438. Same location, $1.3 \% \mathrm{C}, \mathrm{A}_{\mathrm{h}} \mathrm{A}_{1}, 25$ to $45 \mathrm{~cm}$ $3390 \pm 80$ 1440 в.C.

$4020 \pm 70$

BONN-439. Same location, $0.7 \% \mathrm{C}, \mathrm{B}_{\mathrm{th}}, 50$ to $70 \mathrm{~cm}$ 2070 в.c.

BONN-440. Same location, $0.6 \% \mathrm{C}, \mathrm{B}_{\mathrm{t}} / \mathrm{C}, 80$ to $90 \mathrm{~cm}$ $4150 \pm 90$ 2200 в.C.

BONN-441. Boehmen, chernozem lessivé, Griserde $4020 \pm 60$ from loess, Smiuce, Hradec Kralové Dist., 2070 B.c. Tilery ( $50^{\circ} 15^{\prime} \mathrm{N}$ Lat, $15^{\circ} 23^{\prime} \mathrm{E}$ Long), $0.5 \% \mathrm{C}, \mathrm{B}_{\text {th }} \mathrm{C}, 80$ to $90 \mathrm{~cm}$

BONN-442. Boehmen, typic chernozem from loess, Brazdim, Prahoviphod Dist. Tilery, $\left(50^{\circ} 11^{\prime} \mathrm{N}\right.$ Lat, $14^{\circ} 35^{\prime} \mathrm{E}$ Long), $22 \% \mathrm{C}, \mathrm{A}_{\mathrm{p}}, 5$ to $30 \mathrm{~cm}$

$1210 \pm 60$ A.D. 740

BONN-443. Same location, $1.5 \% \mathrm{C}, \mathrm{A}_{\mathrm{h}}, 35$ to $50 \mathrm{~cm}$ $2260 \pm 70$ 310 B.C. $3430 \pm 65$

BONN-444. Same location, $1.4 \% \mathrm{C}, \mathrm{A}_{\mathrm{h}} / \mathrm{C}_{\mathrm{Ca}}, 55$ to $65 \mathrm{~cm} \quad 1480$ B.c.

BONN-445. Boehman, Smonitza (vertisol) from tertiary $2050 \pm 70$ marly clay, Prunevor, Choumtov Dist., 100 B.c. slightly slopy, $\left(50^{\circ} 24^{\prime} \mathrm{N}\right.$ Lat, $13^{\circ} 17^{\prime} \mathrm{E}$ Long), $3.7 \% \mathrm{C}, \mathrm{A}_{\mathrm{h}}, 5$ to $30 \mathrm{~cm}$

BONN-446. Same location, $1.7 \% \mathrm{C}, \mathrm{A}_{\mathrm{h}}, 35$ to $50 \mathrm{~cm} \quad \begin{array}{ll}3800 \pm 8 & 1850 \text { B.C. }\end{array}$

BONN-447. Same location, $1.6 \% \mathrm{C}, \mathrm{A}_{\mathrm{h}} / \mathrm{C}_{\mathrm{Ca}}, 55$ to $65 \mathrm{~cm} \quad 4420$ B.c.

BONN-485. Chernozem from Cretaceous marl, $\quad 118.9 \pm 0.3 \%$ Zezelice I, Königrätz Dist.

Modern $\left(50^{\circ} 8^{\prime} \mathrm{N}\right.$ Lat, $15^{\circ} 21^{\prime} \mathrm{E}$ Long) $1.8 \% \mathrm{C}, \mathrm{A}_{\mathrm{h}}, 0$ to $30 \mathrm{~cm}$

BONN-486. Same location, $1.1 \% \mathrm{C}, \mathrm{A}_{\mathrm{h}}, 30$ to $45 \mathrm{~cm} \quad$ A.D. 830

$$
1460 \pm 110
$$

BONN-487. Same location, $0.5 \% \mathrm{C}, \mathrm{A} / \mathrm{C}_{\mathrm{c}}, 45$ to $60 \mathrm{~cm}$ A.D. 490

BONN-488. Pseudogley chernozem from cretaceous $550 \pm 60$ marl Zozelice II, Königrätz Dist. $\left(50^{\circ} 8^{\prime} \mathrm{N}\right.$ Lat, $15^{\circ} 21^{\prime}$ E Long) $2.1 \% \mathrm{C}, \mathrm{A}_{\mathrm{h}}, 0$ to $30 \mathrm{~cm}$ 
BONN-489. Same location, $1.5 \% \mathrm{C}, \mathrm{A}_{\mathrm{h}}, 35$ to $50 \mathrm{~cm} \quad 1950 \pm 70$

BONN-490. Same location, $1.1 \% \mathrm{C}, \mathrm{A}_{\mathrm{h}} / \mathrm{C}_{\mathrm{c}}, 50$ to $60 \mathrm{~cm}$ A.D. 680

$$
1270 \pm 6 \check{5}
$$

BONN-491. Chernozem-Griserde from loess, lessivé Maehren, Brnicko, Olmütz Dist.

$1080 \pm 65$ (49 $47^{\prime} \mathrm{N}$ Lat, $17^{\circ} 7^{\prime} \mathrm{E}$ Long)

A.D. 870 $1.8 \% \mathrm{C}, \mathrm{A}_{\mathrm{p}}, 0$ to $20 \mathrm{~cm}$

BONN-492. Same location, $1.6 \% \mathrm{C}, \mathrm{A}_{\mathrm{h}} / \mathrm{A}_{1}, 20$ to $35 \mathrm{~cm}$ A.D. 540

$$
1410 \pm 65
$$

BONN-493. Same location, $1.4 \% \mathrm{C}, \mathrm{B}_{\text {th } 1}, 35$ to $52 \mathrm{~cm}$ $3130 \pm 75$ 1180 B.C.

BONN-494. Same location, $1.1 \% \mathrm{C}, \mathrm{B}_{\text {th }}, 52$ to $62 \mathrm{~cm}$

$\begin{array}{lll} & & 4055 \pm 80 \\ \text { BONN-495. Same location, } 0.8 \% \mathrm{C}, \mathrm{B}_{\mathrm{t}} / \mathrm{C}, 62 \text { to } 72 \mathrm{~cm} & 2105 \text { B.c. }\end{array}$

BONN-496. Chernozem from sand loess, Maehren ( $48^{\circ} 45^{\prime} \mathrm{N}$ Lat, $16^{\circ} 53^{\prime} \mathrm{E}$ Long) $1.3 \% \mathrm{C}, \mathrm{A}_{\mathrm{p}}, 0$ to $25 \mathrm{~cm}$

A.D. 1510

BONN-497. Same location, $1.2 \% \mathrm{C}, \mathrm{A}_{\mathrm{l}}, 25$ to $45 \mathrm{~cm}$

$$
1560 \pm 60
$$

A.D. 390

$3610 \pm 75$

BONN-498. Same location, $0.6 \% \mathrm{C}, \mathrm{A}_{\mathrm{h}} / \mathrm{C}, 45$ to $55 \mathrm{~cm} \quad 1660$ B.C.

BONN-499. Same location, $0.5 \%$ C, A $/$ C, 55 to $65 \mathrm{~cm} \quad 3210 \pm 75$

BONN-500. Chernozem from loess, typic, Maehren, $\quad 450 \pm 60$ Bilorice, Bilorice Dist. $\left(48^{\circ} 51^{\prime} \mathrm{N}\right.$ Lat, $16^{\circ} 54^{\prime} \mathrm{E}$ Long) A.D. 1500 $2.3 \% \mathrm{C}, \mathrm{A}_{\mathrm{p}}, 0$ to $25 \mathrm{~cm}$

BONN-601. Same location, $2.6 \% \mathrm{C}, \mathrm{A}_{\mathrm{h}}, 25$ to $40 \mathrm{~cm} \quad$ A.D. 340

BONN-602. Same location, $1.3 \% \mathrm{C}, \mathrm{A}_{\mathrm{h}} / \mathrm{C}_{\mathrm{c}}, 40$ to $55 \mathrm{~cm}$ A.p. 250

$$
1700 \pm 65
$$

$$
2450 \pm 70
$$

BONN-603. Same location, $0.7 \% \mathrm{C}, \mathrm{C}_{\mathrm{c}}, 55$ to $65 \mathrm{~cm}$ 500 B.c.

BONN-604. Chernozem, vertisol-like, Tegel Maehren, Pole, Brünn-Dist.

$117.4 \pm 0.8 \%$ $\left(49^{\circ} 14^{\prime} \mathrm{N}\right.$ Lat, $16^{\circ} 37^{\prime}$ E Long) Modern $2.9 \% \mathrm{C}, \mathrm{A}_{\mathrm{p}}, 0$ to $35 \mathrm{~cm}$ 
BONN-605. Same location, $1.7 \% \mathrm{C}, \mathrm{A}_{\mathrm{h}, \mathrm{c}}, 35$ to $50 \mathrm{~cm}$ $2940 \pm 65$ 990 B.c.

BONN-606. Same location, $1.5 \% \mathrm{C}, \mathrm{A}_{\mathrm{h}, \mathrm{c}}, 50$ to $70 \mathrm{~cm}$ $3690 \pm 70$ 1740 B.c. $4070 \pm 70$

BONN-607. Same location, $0.9 \% \mathrm{C}, \mathrm{A} / \mathrm{C}_{\mathrm{c}}, 70$ to $80 \mathrm{~cm}$ 2120 B.C.

Samples BONN-437-447 as well as BONN-485-500 and BONN-601607 from various great soil groups of Udolls in plains and slightly rolling areas of Czechoslovakia. Coll. and subm. 1968 by D. Nemécek Sec. of Soil Sci., Research Center for Plant Prod., Prague. Comment: except for vertisol-like chernozem (BONN-447) maximum age in deepest layers of A horizons lags behind maximum ages found in $\mathrm{N}$ boundary area of Western German "Feuchtschwarzerden", Brunswick region (BONN-32, BONN-105, BONN-113), but comply very well with maximum ages obtained for Hildesheim pseudogley chernozems (BONN-119, BONN-127). Vertisol-like profile, Tegel, (BONN-604-607) shows fairly low age gradient, reflecting homogenizing effect of longterm recycling self-mulching principle, which is an intrinsic property of these soils. Work is in progress on buried fossil horizons of Czechoslovakia, that merge elsewhere into recent profile, to produce correction factor for rejuvenation ( $c f$. BONN403-416).

Deep plowed degraded chernozem on young Würm Loess WIII, Florsheim (Hessen) on middle terrace of Main $\mathrm{R}$.

BONN-453. Florsheim (Hessen), 0.6\% C, RM, 30 to $40 \mathrm{~cm}$

BONN-454. Same location, $0.4 \% \mathrm{C}, \mathrm{A}_{\mathrm{h}}, 50$ to $60 \mathrm{~cm}$

$$
\begin{aligned}
& 990 \pm 40 \\
& \text { A.D. } 960 \\
& 3360 \pm 80 \\
& 1410 \text { B.C. }
\end{aligned}
$$

Samples coll. 1968 and subm. by H. Zakosek, Hessisches Landesamt für Bodenforschung, Wiesbaden (50 $17.5^{\prime} \mathrm{N}$ Lat, $8^{\circ} 58^{\prime} \mathrm{E}$ Long).

Comment: age of deeper sample is about average so far measured at this depth in chernozem samples of Germany. loess.

Several forms of Russian chernozem (hapl-, vermudoll) on Würm

BONN-455. Deep chernozem from loess, Orel (52.5 $5^{\circ} \mathrm{N}$ Lat, $36.2^{\circ} \mathrm{E}$ Long) $4.5 \% \mathrm{C}, \mathrm{A}_{\mathrm{p}}, 10$ to $20 \mathrm{~cm}$

BONN-456. Same location, $2.3 \% \mathrm{C}, \mathrm{A}_{\mathrm{h}}, 50$ to $60 \mathrm{~cm}$

BONN-457. Same location, $1.0 \% \mathrm{C}, \mathrm{AC}, 110$ to $120 \mathrm{~cm}$

$$
\begin{gathered}
1020 \pm 70 \\
\text { A.D. } 930 \\
2680 \pm 70 \\
730 \text { B.C. }
\end{gathered}
$$

$4720 \pm 60$ 2770 B.C. 
BONN-460. Typical chernozem from loess, Charkov $\left(50^{\circ} \mathrm{N}\right.$ Lat, $36^{\circ} 12^{\prime} \mathrm{E}$ Long) $3.3 \% \mathrm{C}, \mathrm{A}_{\mathrm{p}}, 10$ to $20 \mathrm{~cm}$

BONN-461. Same location, $2.8 \% \mathrm{C}, \mathrm{A}_{\mathrm{h}}, 50$ to $60 \mathrm{~cm}$

BONN-462. Same location, $0.7 \% \mathrm{C}, \mathrm{AC}, 110$ to $120 \mathrm{~cm}$

BONN-464. S chernozem from loess, Zaparoskje $\left(49^{\circ} \mathrm{N}\right.$ Lat, $35^{\circ} \mathrm{E}$ Long) $2.0 \% \mathrm{C}, \mathrm{A}_{\mathrm{p}}, 10$ to $20 \mathrm{~cm}$

BONN-466. Same location, $1.0 \% \mathrm{C}, \mathrm{AC}, 110$ to $120 \mathrm{~cm}$

BONN-468. Chestnut soil from loess, Askania Nova ( $46^{\circ} 30^{\prime} \mathrm{N}$ Lat, $34^{\circ} \mathrm{E}$ Long) $2.0 \% \mathrm{C}, \mathrm{A}_{\mathrm{p}}, 10$ to $20 \mathrm{~cm}$
$1190 \pm 60$ A.D. 760

$$
2650 \pm 70
$$

700 в.C.

$5920 \pm 140$

3970 B.C.

A.D. 1010

$940 \pm 90$

$$
3270 \pm 80
$$

1320 B.c.

$$
1010 \pm 60
$$$$
\text { A.D. } 940
$$

$$
1580 \pm 90
$$

A.D. 370

$$
2710 \pm 70
$$

BONN-470. Same location, $0.7 \% \mathrm{C}, \mathrm{AC}, 110$ to $120 \mathrm{~cm} \quad 760$ в.C.

Samples coll. 1967 and subm. by H. Zakosek. Comment: although Russian chernozems have developed deeper A horizons, age of these samples is about the same as in deepest humus layer of West German chernozems (BONN-105, -112) indicating similar period of origin (Scharpenseel and Pietig, $1969 \mathrm{~b}$ ).

\section{B. Vertisol}

Deepest humus containing layer of Tunesian Vertisols.

\section{BONN-433. Vertisol, Béja}

$2920 \pm 40$

970 B.c.

$\left(36^{\circ} 55^{\prime} \mathrm{N}\right.$ Lat, $8^{\circ} 39^{\prime} \mathrm{E}$ Long) $0.5 \% \mathrm{C}, \mathrm{AC}, 60$ to $85 \mathrm{~cm}$. Slightly vertic dark xerert soil on calcareous loam, $2 \mathrm{~km} \mathrm{~W}$ Béja. Coll. 1968 and subm. by H. W. Scharpenseel.

\section{BONN-434. Vertisol Zouarine}

Near Ebba Ksour (35 59' N Lat, $8^{\circ} 49^{\prime}$ E Long) $0.6 \%$ C, AC, 140 to $170 \mathrm{~cm}$. Grumustert Zouarine, $30 \mathrm{~km} \mathrm{SW}$ Le Kef. Sample taken from maximum penetration depth of cracks. Coll. 1968 and subm. by H. W. Scharpenseel. Comment: both ages of BONN-433 and -434 were expected to be higher. Homogenizing effect of self mulching, vertic principle seems to be cause.

\section{Parabraunerde (hapludalf)} pit.

Parabraunerde (hapludalf) or refilled and recultivated brown coal 
BONN-436. Parabraunerde Bergheim

A.D. 1220

$\left(50^{\circ} 56^{\prime} \mathrm{N}\right.$ Lat, $6^{\circ} 43^{\prime} \mathrm{E}$ Long) $0.3 \% \mathrm{C}, \mathrm{A}_{\mathrm{p}}, 15$ to $25 \mathrm{~cm}$. Sample is mixture of raw loess and former Parabraunerde, flooded by hydraulic transport on top of refilled brown coal pit and re-used for crop production for ca. 10 yr. Coll. 1967 and subm. by E. Schulze, Inst. of Agron., Bonn Univ. Comment: humus produced in $10 \mathrm{yr}$ after recultivation was expected to contain all bomb carbon. Extracts of humic matter were planned to be fractioned for relative age determination of humic matter fractions with reference to bomb carbon distribution curve of last $10 \mathrm{yr}$. Influence of residual humus however too high for fraction dating by bomb carbon measurement.

\section{Buried organic matter}

Organic matter-containing loam for dating age of soil formation along slight grade.

BONN-448. W. Eddersheim, humus loam

$8300 \pm 120$

$\left(50^{\circ} 2^{\prime} \mathrm{N}\right.$ Lat, $8^{\circ} 28^{\prime} \mathrm{E}$ Long) $0.6 \% \mathrm{C}$

Sample from W Eddersheim (Hessen) coll. and subm. by A. Semmel. Comment: BONN-448 complies well with age expectations from 7 to $10,000 \mathrm{yr}$.

Humus-containing sand with charcoal, fireplace.

BONN-608a. Fireplace Amalienhof, $60 \mathrm{~cm}$ deep charcoal only

$2530 \pm 70$

580 в.C.

BONN-608b. Same location humus sand without $\quad 2350 \pm 80$ charcoal, $1.9 \% \mathrm{C}$

400 B.C.

Fireplace Amalienhof, Berlin-Brandenburg (52 $31^{\prime} \mathrm{N}$ Lat, $30^{\circ} 9^{\prime}$ E Long). Coll. and subm. 1968 by U. Schwertmann, Inst. für Bodenkunde, Tech. Univ. Berlin. Comment: fireplace is cut by clay strings. Age determination allows conclusion on clay migration. BONN-608 a, b are less than estimated $4000 \mathrm{yr}$. Pure charcoal is ca. $180 \mathrm{yr}$ older than mixture of humus and charcoal powder.

BONN-609. Buried humus $A_{h}$, Heiligensee Forest, $\quad 760 \pm 60$

$$
0.6 \% \mathrm{C}, 210 \mathrm{~m} \text { A.D. } 1190
$$

Humus under dune sand in Heiligensee Forest, Berlin-Brandenburg $\left(52^{\circ} 36^{\prime} \mathrm{N}\right.$ Lat, $30^{\circ} 56^{\prime} \mathrm{E}$ Long). Coll. and subm. by U. Schwertmann. Comment: humus $210 \mathrm{~m}$ under dune sand. BONN-609 indicates age of dune formation and time span for recent soil development.

Sandy humus, fossil organic matter.
BONN-449. Sandy humus, Kevo, N Finlandia, $\quad 2350 \pm 70$ 75 to $80 \mathrm{~cm}$

Sample from 75 to $80 \mathrm{~cm}$ depth at entrance of seismologic tunnel to Research Sta. Kevo, Finlandia (69 $69^{\circ}$ N Lat, $27^{\circ} 3^{\prime}$ E Long). Coll. and 
subm. 1968 by A. Semmel. Comment: BONN-449 indicates age of soil formation with some rejuvenation by penetrating roots.

Fossil A horizon of humus silty sand.

\section{BONN-432. Fossil A horizon, Spitzbergen, $\quad 3040 \pm 80$ 50 to $60 \mathrm{~cm} \quad 1090$ B.c.}

Fossil horizon at 50 to $60 \mathrm{~cm}$ depth, Hohenstaufen Plateau, Barents I, SE Spitzbergen (no exact coordinates measured). Coll. and subm. 1968 by A. Semmel. Comment: sample indicates age and speed of soil formation under cold climate conditions. BONN-432 agrees with estimates.

Bone relics in loess, Michelsberg.

\section{E. Bones in loess}

\section{BONN-763. Bone-collagen, Michelsberg, 5 m}

$$
\begin{gathered}
10,800 \pm 100 \\
8850 \text { в.C. }
\end{gathered}
$$

Bones in loess deposit under trachyt pumice of Aller $\phi$ d volcanism, $5 \mathrm{~m}$ deep, from Michelsberg, between Ochtendung and Plaidt, Rhineland Pfalz $\left(50^{\circ} 21^{\prime} \mathrm{N}\right.$ Lat, $7^{\circ} 1^{\prime} \mathrm{E}$ Long). Coll. and subm. 1969 by E. Kopp of the Inst. and H. Remy, Inst. of Paleontol., Bonn Univ. Comment: sample of individual bones, mostly from mole, treated with $\mathrm{HCl}$ to separate collagen. Age, 10,800, is younger than expected, since sample originates from loess underlying BONN-413-416. Since bones are in undisturbed position, their later emplacement seems highly improbable.

\section{F. Soil organic matter fractions}

Soil organic matter fractions are dated for information on eventual time sequence of fractions formation. In previous work Münnich, (1957) good agreement was found between total-, cellulose-, and humic mattercarbon. Two samples: St-554 A, St-554 B (Radiocarbon 1963, v. 5, p. 221) showed an age gradient from humic acid via humine and humus coal. When testing decay of young organic masses in soil on basis of bomb carbon levels, Nakhla and Delibrias (1967) found development of humine to occur faster than that of humic acid. Paul et al. (1964), when testing organic matter fractions of chernozem, obtained younger age for fulvic acids, and equal age within error range or humic acid and humine fractions.

BONN-6 A. Söllingen-chernozem, total organic substance $2100 \pm 80$ $\left(52^{\circ} 5^{\prime} \mathrm{N}\right.$ Lat, $10^{\circ} 58.5^{\prime} \mathrm{E}$ Long), AC, 60 to $80 \mathrm{~cm} 150$ B.C.

$\begin{array}{lc}2240 \pm 80 & \pm \text { Same location, only humic acid extract } \\ 290 \text { B.c. }\end{array}$

BONN-138. Podzol Scherpenseel, brown humic acid fraction $2060 \pm 60$ $\left(50^{\circ} 56.5^{\prime} \mathrm{N}\right.$ Lat, $6^{\circ} 0.5^{\prime} \mathrm{E}$ Long), $\mathrm{B}_{\mathrm{h}}, 50$ to $70 \mathrm{~cm} 110$ B.C.

BONN-139. Same location, gray humic acid fraction

$$
1720 \pm 60
$$$$
\text { A.D. } 230
$$ 
BONN-366. Podzol Scherpenseel, fulvic acid fraction, $\mathrm{N}$ rim of gravel pit, $\left(50^{\circ} 56.5^{\prime} \mathrm{N}\right.$ Lat, $6^{\circ}, 0.5^{\prime}$ E Long) $A_{h}, 20$ to $30 \mathrm{~cm}$

BONN-367. Same location, hymatomelanic acid fraction

BONN-368. Same location, brown humic acid fraction

BONN-369. Same location, gray humic acid fraction

BONN-370. Same location, humine fraction

BONN-360. Kalkarer Moor, fulvic acid fraction, near Euskirchen, Rhineland, W marginal area $\left(50^{\circ} 36^{\prime} \mathrm{N}\right.$ Lat, $6^{\circ} 40^{\prime} \mathrm{E}$ Long), $\mathrm{O}$ horizon, $80 \mathrm{~cm}$

BONN-361. Same location, hymatomelanic acid fraction

BONN-362. Same location, brown humic acid fraction

BONN-363. Same location, gray humic acid fraction

BONN-364. Same location, humine fraction

BONN-365. Same location, humus coal fraction

BONN-397. Pseudogley chernozem, fulvic acid fraction, Adlum near Hildesheim, lowest part of $S_{\mathrm{w}} \mathrm{A}, 80 \mathrm{~cm}\left(52^{\circ} 15^{\prime} \mathrm{N}\right.$ Lat, $10^{\circ} 3^{\prime} \mathrm{E}$ Long)

BONN-398. Same location, hymatomelanic acid fraction

BONN-399. Same location, brown + gray humic acid fraction

BONN-401. Same location, humine fraction

BONN-402. Same location, humus coal fraction
$2930 \pm 40$

980 в.C.

$1580 \pm 80$

A.D. 370

$2530 \pm 60$

A.D. 580

$2980 \pm 70$

1030 B.c.

$2850 \pm 70$

900 B.c.

$4270 \pm 80$ 2320 B.C.

$4510 \pm 80$

2560 B.C.

$5380 \pm 80$

3430 B.C.

$5970 \pm 40$

4020 B.C.

$3490 \pm 70$

1540 B.C.

$4460 \pm 80$

2510 B.c.

$1800 \pm 60$ A.D. 150

$1390 \pm 70$ A.D. 560

$4890 \pm 50$ 2940 B.C.

$2980 \pm 70$ 1030 B.C.

$2810 \pm 60$ 860 B.C.

Fractions are taken from pseudogley chernozem, low moor and podzol -organic matter samples. Coll. 1967, fractions separated and subm. by H. W. Scharpenseel and C. Ronzani of the Inst. Comment: BONN-6A 
and BONN-6B, chernozem in total and chernozem humic acid extract are of equal age within error range. From podzol samples BONN-138 and BONN-139, brown humic acid is slightly older than gray humic acid, that occurs in podzol only in scanty amounts and is untypic. Podzol fractions 366-370 are not very different, except for the hymatomelanic acid fraction, that might be contaminated by extraction with modern ethanol. In podzol, interconversions between humic and fulvic acid are most likely occuring. Low moor and pseudogley chernozem fractions show highest age in humic acids. In such profiles, strongly influenced by moisture excess, humine and humus coal fractions are unspecific and contain various residual organic materials of non-humine or non-humus coal character. Fraction results of additional terrestric, non-hydromorphous soil materials are forthcoming.

\section{ARCHAEOLOGIC SAMPLES}

\section{A. West Germany}

BONN-450. Oak wood, Wallerfangen, 3

A.D. 1690

$$
260 \pm 60
$$

A.D. 1740

BONN-451. Same location, 4

$210 \pm 50$

BONN-452. Same location, 5

Modern

BONN-657. Same location, trough-rest of wood in $25 \mathrm{~m}$ deep copper mine

$150 \pm 50$ A.D. 1800

BONN-658. Same location, rest of ladder pole, wood in copper mine

$230 \pm 60$

A.D. 1720

$360 \pm 60$

BONN-659. Same location, wood in copper mine

A.D. 1590

Oak wood, Wallerfangen, Saargebiet, W Germany. Samples found in water, containing copper, in Buntsandstone, St. Barbara village, Blauwald Dist. Continuation of BONN-435 (Radiocarbon, 1969, v. 11, p. 9) $\left(49^{\circ} 22^{\prime} \mathrm{N}\right.$ Lat, $6^{\circ} 43^{\prime} \mathrm{E}$ Long). Coll. and subm. by H. Conrad, Bergbaumus., Bochum. Comment: dates primitive copper mining in this area, "Pingenbau." Expected ages from 1st to 3rd centuries A.D. are ruled out.

\section{B. Cyprus}

Wooden pieces of antique Cypric mine, Cyprus, Apliki.

\section{BONN-677. Wood from Cyprus copper mine}

Cyprus samples ( $35^{\circ} \mathrm{N}$ Lat, $33^{\circ} \mathrm{E}$ Long), nearby Cypric-Roman ceramics found. Coll. 1968 by Kortan, Cyprus Mine Corp., and subm. 
by H. Conrad. Comment: estimated age: 100 to 200 A.D., i.e., 300 to 500 yr younger than BONN-677 and BONN-678.

IV. MODERN SAMPLES

Grass from Röttgen, $8 \mathrm{~km}$ SW Bonn, Rhineland.
BONN-385. Grass, Röttgen, January, 1968
$152.8 \pm 0.6 \%$ Modern
BONN-386. Grass, Röttgen, February, 1968
$152.0 \pm 0.7 \%$ Modern
BONN-387. Grass, Röttgen, March, 1968
$159.0 \pm 0.9 \%$ Modern
BONN-388. Grass, Röttgen, April, 1968
$157.8 \pm 0.9 \%$ Modern
BONN-389. Grass, Röttgen, May, 1968
$150.7 \pm 0.7 \%$ Modern
BONN-390. Grass, Röttgen, June, 1968
$158.7 \pm 0.8 \%$ Modern
BONN-391. Grass, Röttgen, July, 1968
$156.2 \pm 0.6 \%$ Modern
BONN-392. Grass, Röttgen, August, 1968
$161.0 \pm 0.9 \%$ Modern
BONN-393. Grass, Röttgen, September, $1968 \quad 156.0 \pm 0.4 \%$ Modern
BONN-394. Grass, Röttgen, October, $1968 \quad 146.9 \pm 0.7 \%$ Modern
BONN-395. Grass, Röttgen, November, $1968 \quad 146.2 \pm 0.8 \%$ Modern
BONN-396. Grass, Röttgen, December, $1968 \quad 151.1 \pm 0.6 \%$ Modern

Samples were taken monthly to observe fluctuations of bomb carbon level and as extension of bomb carbon-curve (Radiocarbon, 1969, v. 11, p. 13). Samples were taken exclusively from same meadow area within few $\mathrm{m}^{2}\left(50^{\circ} 4 \mathrm{l}^{\prime} \mathrm{N}\right.$ Lat, $7^{\circ} 5.5^{\prime} \mathrm{E}$ Long). Coll. and subm. 1968 by H. W. Scharpenseel. Comment: among fluctuations, highest activity found in August, lowest in October and November.

Date lists:

\section{REFERENCFS}

Bonn I

Bonn II

Stockholm V

Scharpenseel, Pietig, and Tamers, 1968

Scharpenseel, Pietig, and Tamers, 1969 Östlund and Engstrand, 1963

Balke, K. D., 1969, Geothermische und hydrogeologische Untersuchungen in der südlichen Niederrheinischen Bucht. Dissert., Bonn Univ.

Flaig, W., Scheffer, F., and Klamroth, B., 1955, Zur Charakterisierung der Huminsäuren des Bodens: Z. Pflanzenernähr., Düng., Bodenkunde 71, p. 33.

Münnich, K. O., 1957, Erfahrungen mit der ${ }^{14} \mathrm{C}$-Datierung verschiedener Arten von Sedimenten: Verhandlungen der vierten Int. Tagung der Quartärbotaniker, Veröff, Geobotanisches Inst. Rübel b. Zürich, no. 34.

Nakhla, S. M. and Delibrias, G., 1967, Utilisation de carbon-14 d'origine thermonucléaire pour l'étude de la dynamique de carbon dans le sol: I.A.E.A. Conf. radioactive dating and methods of low level counting, proc. Monaco, p. 169.

Östlund, H. G. and Engstrand, L. G., 1963, Stockholm radiocarbon measurements V: Radiocarbon, v. 5, p. 203-227.

Paul, E. A., Campbell, C. A., Rennie, D. A., and McCallum, K. J., 1964, Investigations of the dynamics of soil humus utilizing carbon dating techniques: 8 th internatl. cong. soil science III, proc. Bucharest, p. 201.

Rauterberg, E. and Kremkus, F., 1951, Bestimmung von Gesamt- und alkalilöslichen Huminstoffen im Boden: Z. Pflanzenernähr., Dïng., Bodenkunde, v. 54, p. 240. 
Scharpenseel, H. W. and Pietig, F., 1969a, Radiokohlenstoff- und Tritium-Datierung von Boden und Wasser durch die Benzolmethode: Geoderma, v. 2, p. 273-289.

1969b, Altersbestimmung von Böden durch die Radio-kohlenstoffdatierungsmethode, III, Böden mit $\mathbf{B}_{+}$-Horizont und fossile Schwarzerden: $Z$. Pflanzenernähr., Düng., Bodenkunde, v. 122, p. 145.

Scharpenseel, H. W., Pietig, F., and Tamers, M. A., 1969, University of Bonn natural radiocarbon measurements II, v. 11, p. 3-14.

1968, University of Bonn natural radiocarbon measurements I, v. 10, p. 8-28.

Scharpenseel, H. W., Ronzani, C., and Pietig, F., 1968, Comparative age determination on different humic matter fractions: I.A.E.A. Symposium isotopes and radiation in soil organic matter studies, proc. Vienna, p. 67-73.

Semmel, A., 1968, Studien über den Verlauf jungpleistozäner Formung in Hessen: Frankfurter Geog. Hefte, 45, W. Kramer Pub., Frankfurt.

Strunk-Lichtenberg, G., 1968, Humusuntersuchungen mittels Farbquotienten an archäologischen Objekten aus dem Neolithikum, rep., "Deutsche Forschungsgemeinschaft", 1968.

Tamers, M. A., 1966, Ground water recharge of aquifers as revealed by naturally occurring radiocarbon in Venezuela: Nature, v. 212, p. 489-493.

1967. Radiocarbon ages of ground water in an arid zone unconfined aquifer: Am. Geophys. Union Mon., v. 11, p. 143

Tamers, M. A., Balke, K. D., and Scharpenseel, H. W., 1968, Untersuchungen zur Fliessgeschwindigkeit des Grundwassers durch Bestimmung der Radiokohlenstoffund Tritium- aktivität: I. Teil. Zeitschr. f. Kulturtechnik u. Flurbereinigung, v. 9 , no. 6 , p. $364-380$. 